\title{
Time-domain fitting of battery electrochemical impedance models
}

\author{
S.M.M. Alavi ${ }^{\mathrm{a}}$, C.R. Birkl ${ }^{\mathrm{a}}$, D.A. Howey ${ }^{\mathrm{a}, 1, *}$ \\ ${ }^{a}$ Energy and Power Group, Department of Engineering Science, \\ University of Oxford, Parks Road, Oxford, OX1 3PJ, United Kingdom
}

\begin{abstract}
Electrochemical impedance spectroscopy (EIS) is an effective technique for diagnosing the behaviour of electrochemical devices such as batteries and fuel cells, usually by fitting data to an equivalent circuit model (ECM). The common approach in the laboratory is to measure the impedance spectrum of a cell in the frequency domain using a single sine sweep signal, then fit the ECM parameters in the frequency domain. This paper focuses instead on estimation of the ECM parameters directly from time-domain data. This may be advantageous for parameter estimation in practical applications such as automotive systems including battery-powered vehicles, where the data may be heavily corrupted by noise. The proposed methodology is based on the simplified refined instrumental variable for continuous-time fractional systems method ('srivcf'), provided by the Crone toolbox [1,2], combined with gradient-based optimisation to estimate the order of the fractional term in the ECM. The approach was tested first on synthetic data and then on real data measured from a 26650 lithium-ion iron phosphate cell with low-cost equipment. The resulting Nyquist plots from the time-domain fitted models match the impedance spectrum closely (much more accurately than when a Randles model is assumed), and the fitted parameters as separately determined through a laboratory potentiostat with frequency domain fitting match to within $13 \%$.
\end{abstract}

Keywords: battery, electrochemical impedance spectroscopy, parameter estimation, fractional order system

\section{Introduction}

Electrochemical impedance spectroscopy (EIS) is a useful non-invasive technique for the study of the dynamics and properties of materials and electrochemical systems [3-7] and may also be used to diagnose temperature [8] and state of health [9] of electrochemical cells non-invasively. The impedance of an electrochemical system around some equilibrium point is determined by applying a low amplitude $\mathrm{AC}$ current and measuring its voltage response, or vice versa [10]. This technique is particularly useful for the study of electrochemical systems whose dynamic response consists of a number of closely coupled processes that occur at different rates. In the particular case of a lithium ion (Li-ion) battery, EIS enables the identification and

\footnotetext{
*Corresponding author. Tel.: +44 1865283 476, Fax.: +44 1865273 010, Room 15.10, Holder Building, Department of Engineering Science, University of Oxford, Parks Road, Oxford, OX1 3PJ, United Kingdom

Email addresses: mahdi.alavi@eng.ox.ac.uk (S.M.M. Alavi), christoph.birkl@eng.ox.ac.uk (C.R. Birkl), david.howey@eng.ox.ac.uk (D.A. Howey)

$U R L$ : http://epg.eng.ox.ac.uk/users/david-howey (D.A. Howey)
} 


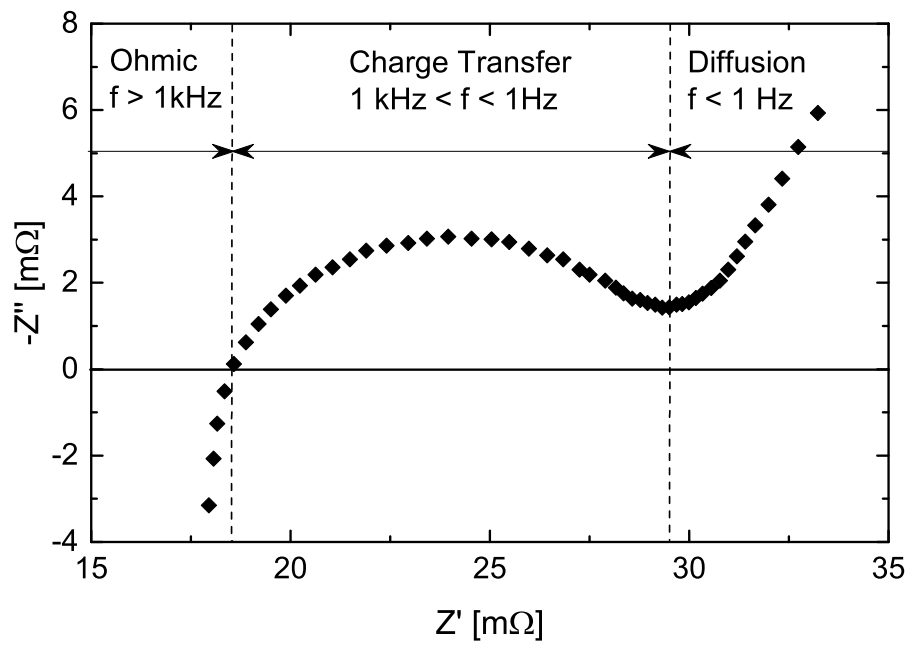

Figure 1: Nyquist plot of impedance spectrum of a Li-ion cell.

study of a number of different processes, including the movement of charge carriers through the electrolyte, current collectors and wires (ohmic resistance at frequencies typically above $1 \mathrm{kHz}$ ), the electrochemical double layer and charge transfer reaction at the electrode surfaces (frequencies between $1 \mathrm{~Hz}$ and $1 \mathrm{kHz}$ ) and the solid state diffusion of lithium ions within the bulk of the electrode material (frequencies usually below 1 $\mathrm{Hz})$ [11]. As an example, Figure 1 shows a Nyquist plot of the impedance spectrum of a $1100 \mathrm{mAh} \mathrm{LiFePO}_{4}$ 18650 cell by A123 Systems, measured with a small signal ac current of $100 \mathrm{~mA}$, in a frequency range of 10 $\mathrm{mHz}$ to $5 \mathrm{kHz}$, at $50 \%$ state of charge (SOC) and ambient temperature. The different processes with typical frequency ranges are annotated in the figure.

For a simplified description of the complex physical and chemical processes occurring within electrochemical systems such as batteries or fuel cells, electrical equivalent circuit models (ECMs) can be used to model the small signal dynamic behaviour $[3,4,12]$. One of the most widely used equivalent circuit representations of Li-ion batteries is based on the Randles circuit [4], which consists of an ohmic resistor to represent the conduction of charge carriers through electrolyte and metallic conductors, and a resistor in parallel with a capacitor to represent the charge transfer resistance and double layer capacitance, respectively. However, these idealised circuit components are inadequate to model electrochemical charge transfer and double layer capacitance in real systems due to the spatial distribution of these processes. For a better representation of distributed electrode processes in real systems, Cole and Cole [3] proposed an equivalent circuit element with constant phase, independent of the frequency: the so-called constant phase element (CPE), which has been adopted for Li-ion ECMs (e.g. [13]). The impedance of the CPE, $Z_{C P E}$, is expressed as:

$$
Z_{C P E}(\omega)=\frac{1}{Q(j \omega)^{\alpha}}
$$




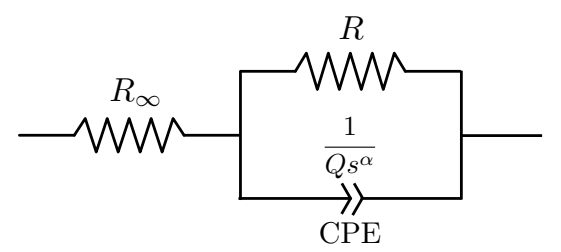

Figure 2: Battery EIS equivalent circuit model (2).

where $Q$ is a constant, $j$ is the imaginary number, $\omega$ is the radian frequency and $\alpha(0>\alpha>1)$ is the exponent value, which is 1 for an ideal capacitor. In the fractional case, the dimension of $Q$ is $\mathrm{Fcm}^{-2} s^{\alpha-1}$ $[14,15]$. In order to model the impedance for all high- to mid-frequency processes in a Li-ion cell, the CPE is combined in parallel with a resistor representing charge transfer resistance $(R)$, and in series with another resistor representing ohmic contributions $\left(R_{\infty}\right)$, which results in:

$$
Z(\omega)=R_{\infty}+\frac{R}{R Q(j \omega)^{\alpha}+1}
$$

where, $Z$ denotes the cell impedance. If we replace $j \omega$ with the Laplace operator $s$ in (2), then the ECM expressed as a transfer function is:

$$
Z(s)=\frac{V(s)}{I(s)}=R_{\infty}+\frac{R}{R Q s^{\alpha}+1}
$$

where $V(s)$ and $I(s)$ are the Laplace transforms of the cell voltage and current signals. Figure 2 shows the equivalent circuit of the EIS model (3).

Although relatively simple, this model is sufficient to represent the basic dynamic behaviour of a large number of electrochemical systems [16]. The model could be extended to include further R-CPE parallel pairs depending on the electrochemical system. To model the Warburg element, a single CPE in series with $R_{\infty}$ could be added. However, in order to demonstrate the time-domain fitting technique without overfitting, we focus on using this model to solve the following problem:

Problem Statement: Consider an electrochemical system with an EIS model given by (3). The problem is to estimate the values of $R_{\infty}, R, Q$ and $\alpha$ from suitably excited current and voltage, $i(t)$ and $v(t)$, such that the frequency response of $Z(s)$ fits the measured impedance spectra within the frequency range of interest $\left[\omega_{L}, \omega_{H}\right]$. It is assumed that $i(t)$ and $v(t)$ are discrete time sampled data with sample time $T_{s}$, from $t=0$ to $t=t_{f}=K T_{s}$ and $K \in \mathbb{Z}^{+}$.

For many applications, the ECM parameters of (3) are typically estimated in the frequency domain by complex non-linear least squares (CNLS) regression $[17,18]$. This approach works well in the lab, since low noise frequency domain measurements are directly available by exciting the cell with a sinusoidal excitation at various frequencies. However, this is impractical for condition monitoring in real applications, since it is relatively slow. Instead, a wide range of frequencies may be used to excite the cell at the same time, for example a pseudo-random binary sequence, broadband noise, or multisine excitation [19], allowing a 
much faster measurement, and the possibility to use passively occurring noise as the excitation source. In this scenario, before fitting, time-domain data must be converted to the frequency domain using Fourier transforms. Since the excitation and response data are often non-periodic, a window function must be used to smooth the spectral estimation, however this introduces a bias to the estimation. It is therefore preferable outside the lab for condition monitoring purposes to estimate the model parameters of an EIS model such as (3) directly from time domain measurements of voltage and current, making use of all available data and without introducing bias.

Direct time domain fitting of (3) is challenging, because the exponent $\alpha$ is typically a non-integer due to the CPE. This is termed a 'fractional order system' and since the Laplace operator $s$ represents differentiation, this is equivalent to a dynamic system with a non-integer derivative operator.

In order to address this challenge, CPEs for Li-ion ECMs are generally either approximated by ideal capacitors [20], or by a series connection of numerous R/C pairs [21-23], or a distribution of relaxation times [24]. The former has found widespread application in ECMs used for battery management systems (BMS) of Li-ion batteries due to its simplicity and computational efficiency [25]. This approach may be sufficient to simulate Li-ion cells under load to moderate degrees of accuracy [26] at low sampling frequencies. However, it does not allow for a precise representation of Li-ion dynamics in both the time and frequency domain over a wide frequency range, which is necessary to maintain the physical significance of the ECM for use in further analyses, e.g. with respect to cell ageing and degradation. Similarly, regarding the latter approach of approximating CPEs with numerous R-C pairs, an increasing number of model parameters reduces their physical meaning and increases the risk of over-fitting. This provides an incentive to use a parameter estimation technique directly for the fractional order system of (3), rather than approximations by increasingly empirical ECM structures. The focus of this paper is to demonstrate in practice such fractional order system identification using real data from an electrochemical cell.

The main contribution of this paper is that it demonstrates, using data directly measured from a battery, that fractional order system identification tools may be used to estimate credible EIS model parameters. This opens up opportunities to use a wide range of passive and active excitation signals and therefore to improve diagnostics in electrochemical systems. In contrast to previous non-parametric time-domain approaches $[19,27]$, we demonstrate direct parametric estimation from the time-domain. It should be noted that the proposed method can be applied to any electrochemical system, such as fuel cells or supercapacitors, having the same EIS model structure.

\section{Fractional system identification}

The goal of fractional system identification is to estimate the parameters of the following system:

$$
\begin{array}{r}
y(t)+a_{1} p^{\alpha_{1}} y(t)+\cdots+a_{n} p^{\alpha_{n}} y(t)= \\
b_{0} p^{\beta_{0}} u(t)+b_{1} p^{\beta_{1}} u(t)+\cdots+b_{m} p^{\beta_{m}} u(t)
\end{array}
$$


where $y(t)$ and $u(t)$ denote output and input signals which are measurable observations and $a_{i}, \alpha_{i}, b_{j}$, and $\beta_{j}$ for $i=1, \cdots, n$ and $j=0, \cdots, m$ are unknown parameters to be estimated. The symbol $p$ is the fractional differentiation operator defined as $p^{\gamma}()=.\frac{d^{\gamma}(.)}{d t^{\gamma}}$. The coefficients $a_{i}$ and $b_{j}$ belong to the set of real numbers $\mathbb{R}$. The exponent values $\alpha_{i}$ and $\beta_{j}$ are non-integer positive numbers $\in \mathbb{R}^{+}$.

Taking the Laplace transform of (4) with the assumption that $u(t)=y(t)=0$ for all $t<0$ gives:

$$
F(s)=\frac{Y(s)}{U(s)}=\frac{b_{m} s^{\beta_{m}}+\cdots+b_{1} s^{\beta_{1}}+b_{0} s^{\beta_{0}}}{a_{n} s^{\alpha_{n}}+\cdots+a_{1} s^{\alpha_{1}}+1}
$$

Definition 1. The transfer function (5) is commensurate of order $\gamma$ if all exponent values $\alpha_{i}$ and $\beta_{j}$ for $i=1, \cdots, n$ and $j=0, \cdots, m$ are multiples of $\gamma$, i.e., $\alpha_{i}=N_{i} \gamma$ and $\beta_{j}=M_{j} \gamma$ such that $N_{i}$ and $M_{j}$ belong to positive integer numbers, [28].

Remark 1. Based on Definition 1, the EIS model (3) is commensurate of order $\alpha$.

Identification of fractional order systems was initiated by Le Lay, Lin and Cois in their PhD theses in [29], [30] and [31], respectively. Various algorithms have been proposed which are based on classical least-squares estimation techniques, with applications to thermal diffusion processes [32] and state-of-charge estimation in batteries [33]. In order to address the impact of the measurement noise on the estimation, state variable filters (SVFs) have been employed. However, it was shown in [34] that this may lead to biased estimation in fractional order systems. To address this, adaptions of the refined version [35] of the instrumental variable method [36] have been developed [37, 38], called the instrumental variable state variable filter (isvsf), and the simplified refined instrumental variable for continuous-time fractional models (srivcf) method. It was shown in [37] that both the isvsf and srivcf result in asymptotically unbiased estimations. However, because the SVFs in the srivcf method are optimally computed in each iteration, the variance of the estimated parameters in the srivcf method is significantly reduced compared to that of parameters estimated using the isvsf method.

It should be noted that both the srivcf and isvsf identification methods only estimate the coefficient vectors $\left(a_{i}\right.$ and $\left.b_{j}\right)$ and assume that the fractional orders are a priori known. In these methods, all fractional differentiation operators $s^{\alpha_{i}}$ or $s^{\beta_{j}}$ in (5) are approximated within the bandwidth of interest by higher-order rational transfer functions, [37]. The approximation of $s^{\gamma}$ within the bandwidth $\left[\omega_{L}, \omega_{H}\right]$ is given by

$$
s^{\gamma} \approx \kappa \prod_{i=1}^{N} \frac{\frac{s}{\omega_{z_{i}}}+1}{\frac{s}{\omega_{p_{i}}}+1}
$$


where the zeros and poles, $\omega_{p_{i}}$ and $\omega_{z_{i}}$, are obtained recursively through the following equations:

$$
\begin{aligned}
& \sigma=\left(\frac{\omega_{H}}{\omega_{L}}\right)^{\gamma} \\
& \eta=\left(\frac{\omega_{H}}{\omega_{L}}\right)^{1-\gamma} \\
& \omega_{z_{1}}=\sqrt{\eta} \omega_{L} \\
& \omega_{p_{i}}=\sigma \omega_{z_{i}}, i=1, \cdots, N \\
& \omega_{z_{i+1}}=\eta \omega_{p_{i}}, i=1, \cdots, N-1
\end{aligned}
$$

and $\kappa$ is chosen to provide unity gain at $s=1$.

In the case of unknown fractional orders, additional computational steps must be taken to identify the fractional orders. In [37], a heuristic algorithm was proposed, searching for the commensurate fractional order within a predefined range that minimises the log-normalised error between the actual and estimation outputs. In [38], an alternative gradient-based approach was introduced for the automatic identification of the unknown fractional orders, applicable to both commensurate and non-commensurate systems. Here we combine a constrained minimisation algorithm to estimate the value of $\alpha$ with the srivcf identification algorithm from the CRONE toolbox [1,2] to estimate $R_{\infty}, R$ and $Q$ in (3).

\subsection{Simplified Refined Instrumental Variable for Continuous-time Fractional (srivcf) identification method}

The srivcf algorithm is available in the CRONE toolbox [1,2] and is fully described in [38]. The srivcf method is an extension of the srivc algorithm [39] to fractional order systems. We repeat here only very brief details for clarity. By defining $A(p)=1+\sum_{i=1}^{n} a_{i} p^{\alpha_{i}}$ and $B(p)=\sum_{j=0}^{m} b_{j} p^{\beta_{j}}$, recalling that $p$ is the fractional differentiation operator, the fractional system (4) is written in the following form:

$$
y(t)=\frac{B(p)}{A(p)} u(t)
$$

It is assumed that the output $y(t)$ is corrupted by zero-mean additive noise $\xi(t)$, giving the measured output:

$$
y^{*}(t)=y(t)+\xi(t)
$$

The srivcf algorithm estimates the parameter vector $\theta$ defined as:

$$
\theta=\left[\begin{array}{llllllll}
b_{0} & b_{1} & \cdots & b_{m} & a_{1} & a_{2} & \cdots & a_{n}
\end{array}\right]^{T}
$$

from measurements $u(t)$ and $y^{*}(t)$ with the assumption that all fractional orders are a priori known.

The error to minimise is the difference between the predicted and measured systems, given by the output 
error:

$$
\varepsilon(t, \theta)=y^{*}(t)-\frac{B(p, \theta)}{A(p, \theta)} u(t)
$$

By filtering the input and output signals $u(t)$ and $y^{*}(t)$ using the following low-pass filter,

$$
F(p)=\frac{1}{A(p, \theta)}
$$

the output error (9) is converted into:

$$
\varepsilon(t, \theta)=A(p, \theta) y_{f}^{*}(t)-B(p, \theta) u_{f}(t)
$$

where, $u_{f}(t)=F(p) u(t)$ and $y_{f}^{*}(t)=F(p) y^{*}(t)$ are the filtered input and output respectively. The srivcf algorithm minimises the output error (11) by using a least-squares method. The filter $F(p)$ is unknown and therefore computed iteratively alongside the least squares estimation of the model parameter $\theta$.

The srivcf algorithm can be initialised by using the least-squares SVF or the isvsf method. The asymptotic unbiased convergence of the srivcf method has been proven in [37] if the dimension and commensurate order of the system are known.

\subsection{Identification of the fractional order by constrained minimisation}

In order to identify the fractional order $\alpha$ in (3), we combine a constrained minimisation algorithm (based on Matlab's fmincon) with srivcf. The cost function to minimise is the $\ell_{2}$-norm of the normalised error between the model predicted output and measured output [38]:

$$
J=10 \log _{10}\left(\frac{\sum_{k=0}^{K}\left(\hat{y}\left(k T_{s}\right)-y^{*}\left(k T_{s}\right)\right)^{2}}{\sum_{k=0}^{k=K}\left(\hat{y}\left(k T_{s}\right)\right)^{2}}\right)
$$

where $\hat{y}$ is the model estimated output and $y^{*}$ is the measured output. The optimisation problem is therefore:

$$
\begin{array}{ll}
\operatorname{minimize} & J(\alpha) \\
\text { subject to } & 0 \leq \alpha \leq 1
\end{array}
$$

\subsection{The fitting Algorithm}

Based on the above statements, the EIS identification algorithm is as follows:

\section{Automatic combined srivcf/order-identification EIS time fitting algorithm}

Step 1: Load time domain data, pre-filter if necessary.

Establish initial guesses $R^{0}, R_{\infty}^{0}, Q^{0}, \alpha^{0}$. 


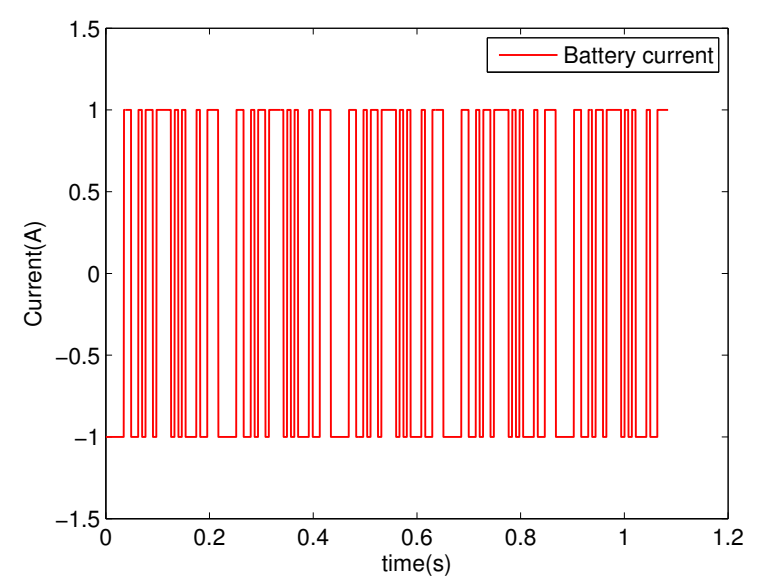

(a) Battery current $u(t)(\mathrm{A})$.

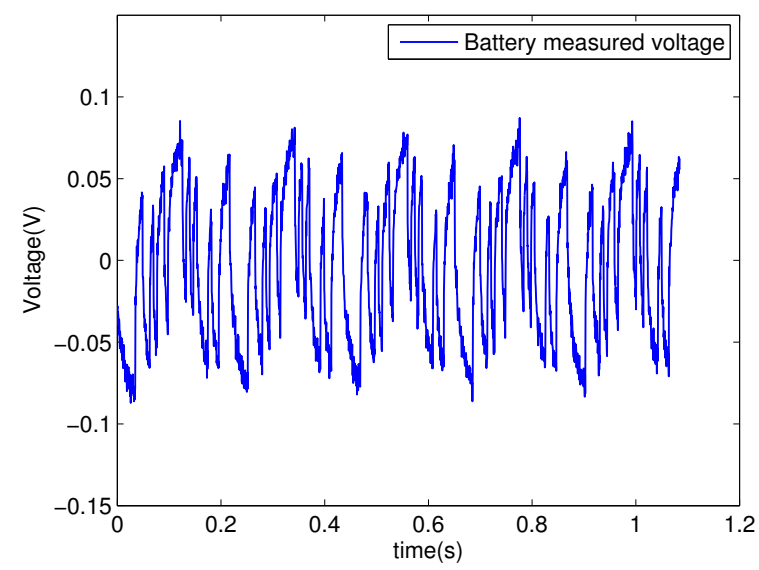

(b) Battery measured voltage $y^{*}(t)(\mathrm{V})$.

Figure 3: Evaluation of the proposed algorithm using synthetic data: measurement signals.

Step 2: Set up minimisation problem including bounds for $\alpha$ and a handle to the cost function which calls srivcf at each iteration.

Step 3: Run fmincon of $\alpha$ until the required tolerance is achieved.

Step 4: Extract the estimated parameters from the fitted fractional transfer function.

\section{End}

\section{Results and discussion}

\subsection{Parameter estimation using synthetic data}

The effectiveness of the proposed fitting algorithm was evaluated first by using synthetic data. Consider the EIS model (3) with true parameters $R_{\infty}=0.018 \Omega, R=0.11 \Omega, Q=1 \mathrm{Fcm}^{-2} s^{-.35}$ and $\alpha=0.65$. The battery current input $u(t)$ is a zero mean pseudo-random binary sequence (PRBS) which is injected into the model, with these parameter values to find the voltage response $y(t)$. The battery voltage is further corrupted by zero-mean white noise with standard deviation $5 \mathrm{mV}$ to give the observed voltage $y^{*}(t)$. Figure 3 shows $u(t)$ and $y^{*}(t)$. It should be noted that the battery nominal voltage has been removed from the data, since this is a small signal identification method.

Remark 2. The quantity and quality of data are very important in the performance of the identification. Practical identifiability analysis [40] is an approach to assess the data. Recently, there has been significant interest in the practical identifiability analysis of battery models (see for instance [41] and references therein), which are mainly based on the Fisher information matrix [42, 43]. In this paper, it is assumed that rich data, from both the quality and quantity perspectives, is available for the identification. 


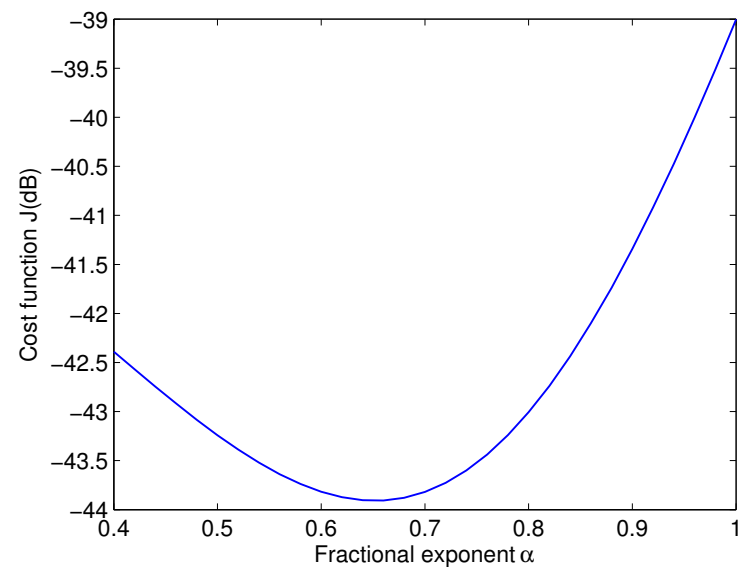

Figure 4: Evaluation of the proposed algorithm using synthetic data: the cost function $J$ given by (12) versus $\alpha$. The minimum $J$ is obtained at the best estimation of the fractional exponent $\alpha$.

Table 1: True vs. estimated parameters using synthetic data

\begin{tabular}{|l|l|ll|}
\hline Parameter & True value & \multicolumn{2}{|c|}{ Estimated value } \\
& & Fractional model Eq. (3) & Randles model Eq. (14) \\
\hline$R_{\infty}$ & $18.0 \mathrm{~m} \Omega$ & $18.3 \mathrm{~m} \Omega$ & $28.6 \mathrm{~m} \Omega$ \\
$R$ & $110.0 \mathrm{~m} \Omega$ & $108.6 \mathrm{~m} \Omega$ & $55.2 \mathrm{~m} \Omega$ \\
$Q(C$ for Randles $)$ & $1.0 \mathrm{Fcm}^{-2} s^{-.35}$ & $1.0 \mathrm{Fcm}^{-2} s^{-.348}$ & $0.25 \mathrm{~F}$ \\
$\alpha$ & 0.65 & 0.652 & 1 \\
\hline
\end{tabular}

The fmincon-srivcf algorithm was applied assuming that $\alpha$ is within the range of $[0.3,1]$ with an initial guess of $\alpha=0.4$ and tolerances of $1 \mathrm{e}-4$ on $\alpha$ and $1 \mathrm{e}-3$ on $J$. The default interior point optimisation algorithm [44] was chosen. The frequency range of interest was $[1,300] \mathrm{Hz}$. The sampling frequency is chosen as $f_{s}=2 \mathrm{kHz}$. With respect to the time duration in Figure 3 , the data length is approximately $2000 \times 1.1 \approx 2200$. Due to the convex cost function (Figure 4), the algorithm converged repeatably to a solution quickly in around 6 iterations, with 15 function calls to srivcf, finding $J_{\min }=-43.91 \mathrm{~dB}$. The minimum $J$ is obtained at the best estimation of the fractional exponent at $\alpha=0.652$. Table 1 compares the true and estimated parameters. Figure 5 shows the voltage response and impedance spectra for both the true and estimated models. The results confirm that the EIS model parameters and also impedance spectra are accurately estimated by using the proposed technique and fractional model (3).

For comparison, a Randles model of the battery is estimated with an ideal capacitor in the form of:

$$
Z_{\text {Randles }}(s)=R_{\infty}+\frac{R}{R C s+1}
$$

using the same identification method. The estimation is performed by fixing the exponent value at 1 . The results are shown in Table 1 and Figure 5. Despite that the voltage response was fitted accurately (Figure $5(\mathrm{a})$ ), the Randles model's parameters are estimated numerically quite far from the true values. As the 


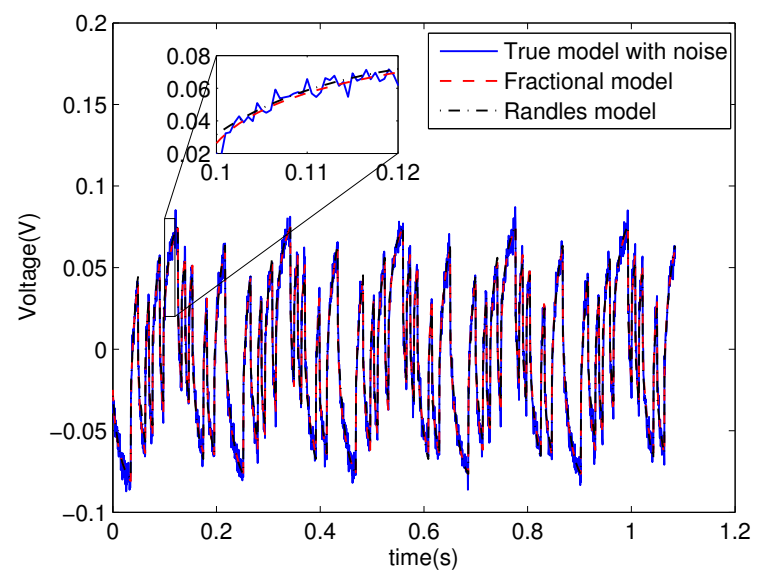

(a) Voltage response.

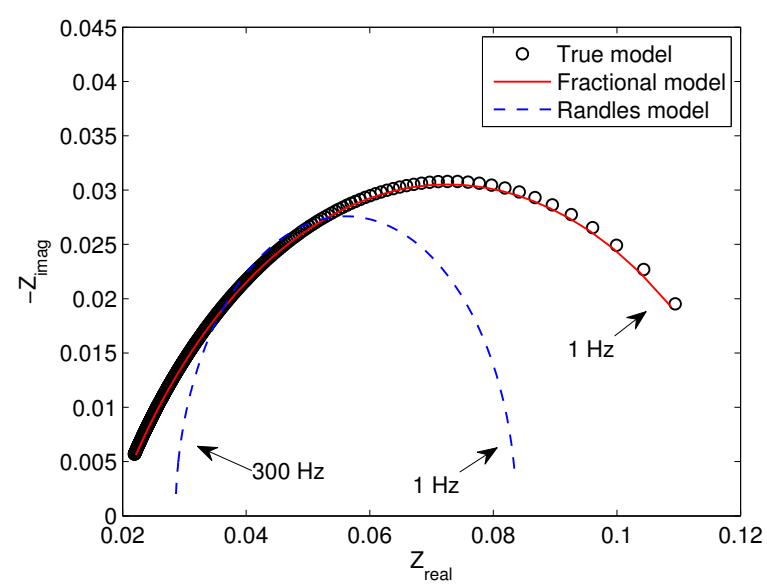

(b) Impedance spectra.

Figure 5: Evaluation of the fractional and Randles models using the synthetic data given in Table 1.

data in Table 1 show, the estimation error of the Randles model is up to $75 \%$. The Nyquist diagram of the Randles model's parameters is a semicircle (with a radius of around 0.025 2 ). Therefore, only a part of the true impedance spectra can be retrieved, and the error is significant at lower and higher frequencies as shown in Figure 5(b).

\subsection{Parameter estimation using real data}

The effectiveness of the algorithm was then evaluated by using real data. The same procedure as in [19] was followed to collect the battery current and voltage, briefly outlined here. The schematic diagram of the measurement circuit is shown in Figure 6. An A123 Systems lithium-ion iron phosphate (LFP) cell was tested, having length $65 \mathrm{~mm}$, diameter $26 \mathrm{~mm}$, nominal capacity $2.3 \mathrm{Ah}$ and nominal voltage $3.3 \mathrm{~V}$. The cell was connected to a TTi electronic load which was controlled by an analog control voltage from a National Instruments NI 6211 ADC/DAC board. The voltage across the cell was measured by a differential amplifier with gain of 1 , then filtered by a passive $\mathrm{RC}$ high pass filter $(\mathrm{C} 1$ and $\mathrm{R} 1$ in Figure 6 , with $-3 \mathrm{~dB}$ point at $1 \mathrm{~Hz}$ ), then amplified by a non-inverting amplifier with a gain of 100 , before analog to digital conversion in the NI 6211 . The current was measured by passing it through a $0.1 \Omega$ precision shunt resistor and measuring the voltage across the resistor with a differential amplifier followed by an RC filter ( $\mathrm{C} 2$ and $\mathrm{R} 4)$, which was then amplified in a non-inverting amplifier and converted from analog to digital, in the same way as the voltage channel. In this case, rather than PRBS, a multisine excitation current signal $u(t)$ comprising 80 logarithmically spaced frequencies from $1 \mathrm{~Hz}$ to $2 \mathrm{kHz}$ was applied, with constant offset $u_{c}$ and weighting $W$ :

$$
u(t)=u_{c}+W \sum_{i=1}^{80} \sin \omega_{i} t
$$

Due to the requirement for uni-directional current flow because the load can only sink current, the multisine excitation current was superimposed on a constant DC offset current $u_{c}$ set to $150 \mathrm{~mA}$ in this experiment. 


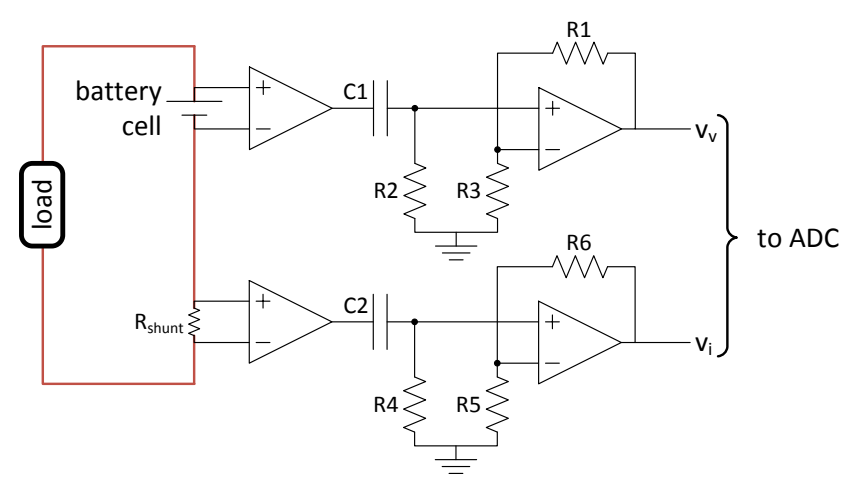

Figure 6: Diagram of voltage and current measurement circuit.

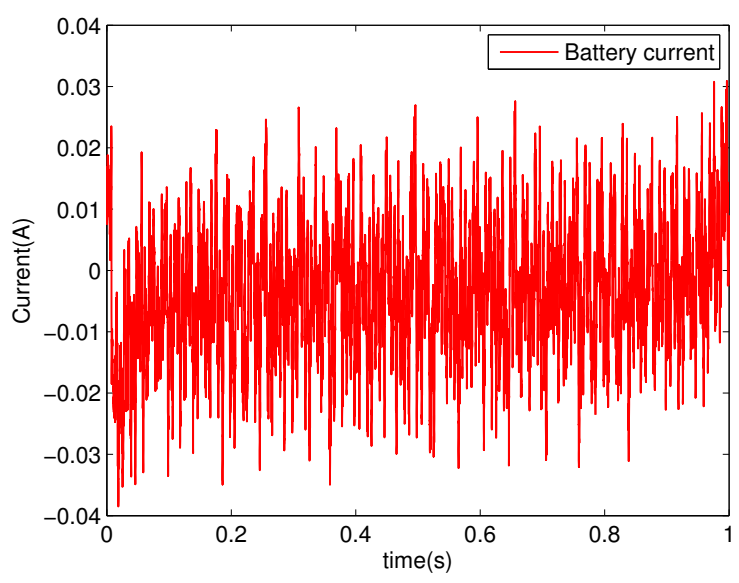

(a) Battery current (A).

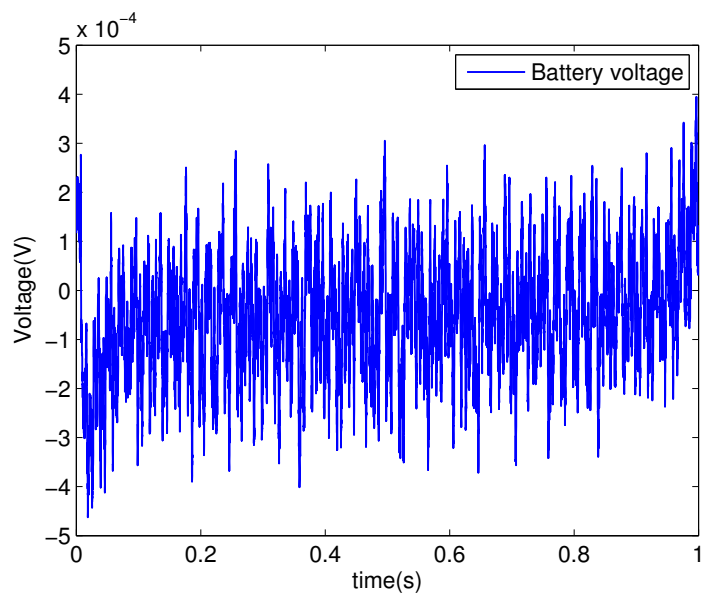

(b) Battery voltage $y^{*}(t)(\mathrm{V})$.

Figure 7: Evaluation of the proposed algorithm using real data: filtered signals.

The peak-to-peak value of the superimposed multisine signal was set to $130 \mathrm{~mA}$ maximum. The sampling frequency was set to $f_{s}=20 \mathrm{kHz}$.

For validation purposes, the impedance spectrum was independently measured directly after the time domain measurement using a Biologic VSP potentiostat using a single-sine sweep method across the same range of frequencies, with the same $\mathrm{AC}$ and $\mathrm{DC}$ currents. This spectrum was then independently fitted to the same ECM structure using the commercial software EC-Lab [45] that uses the simplex algorithm for CNLS optimisation in the frequency domain, with randomised initial parameter values.

In order to apply the proposed time-domain fitting algorithm, the measured current and voltage must be digitally filtered to remove the impact of the measurement circuits in Figure 6, as described in [19]. The filtered current and voltage used for identification are shown in Figure 7. It should be noted that the DC current offset has been removed from the data to give small signal excitations around a zero mean, as usual in linear small signal model identification methods. The time duration is $t_{f}=1 \mathrm{~s}$, therefore, the data length is $20000 \times 1=20000$.

The fitting algorithm was applied in the same way as for the synthetic data previously described. It was 
Table 2: True vs. estimated parameters using real data

\begin{tabular}{|l|l|ll|}
\hline Parameter & Frequency domain & \multicolumn{2}{|c|}{ Time domain estimation value } \\
& estimation value & Fractional model Eq. (3) & Randles model Eq. (14) \\
\hline$R_{\infty}$ & $8.31 \mathrm{~m} \Omega$ & $7.40 \mathrm{~m} \Omega$ & $9.4 \mathrm{~m} \Omega$ \\
$R$ & $6.66 \mathrm{~m} \Omega$ & $7.50 \mathrm{~m} \Omega$ & $4.7 \mathrm{~m} \Omega$ \\
$Q(C$ for Randles $)$ & $2.32 \mathrm{Fcm}^{-2} s^{-.29}$ & $2.04 \mathrm{Fcm}^{-2} s^{-.36}$ & $0.45 \mathrm{~F}$ \\
$\alpha$ & 0.71 & 0.64 & 1 \\
\hline
\end{tabular}

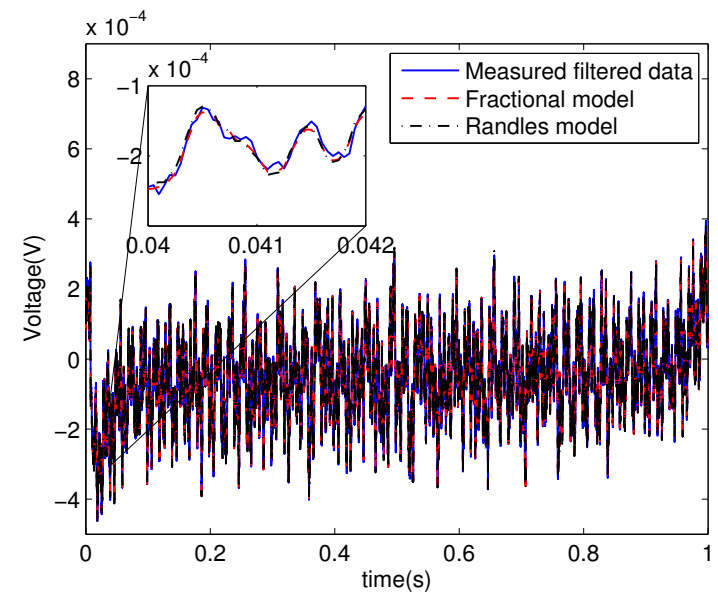

(a) Voltage response.

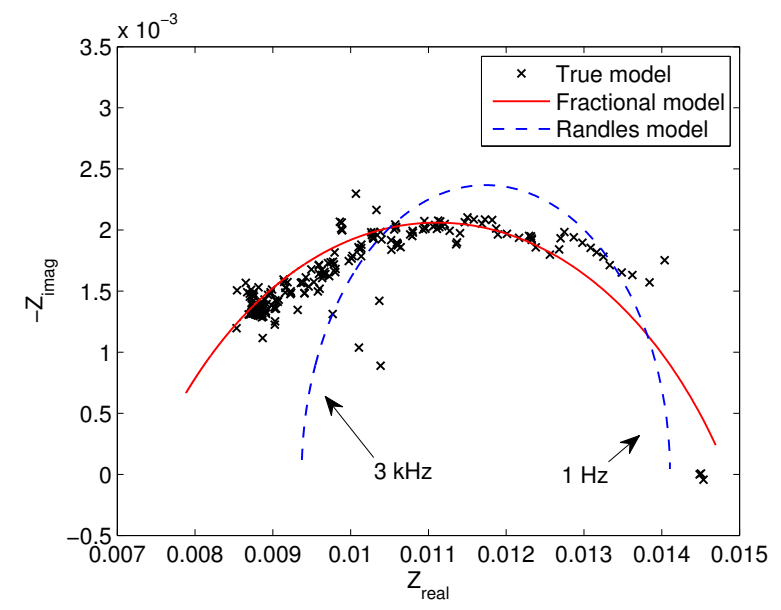

(b) Impedance spectra.

Figure 8: Evaluation of the fractional and Randles models using the real data given in Table 2.

assumed that $\alpha$ was within the range $[0.3,1]$. The minimum and maximum frequencies of the multisine signal are $1 \mathrm{~Hz}$ and $2 \mathrm{kHz}$ respectively, so the frequency range of interest was chosen to be [0.7, 3000] Hz in order to give some frequency range above the maximum and below the minimum applied excitation frequencies. The fmincon-srivef algorithm converged to a minimum with cost function equal to $J_{\min }=-55.57 \mathrm{~dB}$. The shape of $J$ versus $\alpha$ is smooth and convex smilier to that of the synthetic data, therefore the figure is not shown. Table 2 compares the estimated parameters from the time domain method to the parameters estimated from the separate measurement and frequency domain fitting made with the Biologic VSP and EC-Lab software. As can be seen all parameters are within $13 \%$ of those measured independently. The discrepancy between $R_{\infty}$ values may be caused by the disconnecting and reconnecting of the cells between the independent tests, and the small discrepancies in the other values may be caused by slight differences in low frequency behaviour due to diffusion, which is not captured in the ECM structure. Figure 8(a) compares the model voltage response with the measurement response in the time domain. Figure 8(b) shows the frequency domain fit between the model frequency response and the real data at the frequencies of highest coherence. Both results are quite satisfactory.

Again, a Randles model of the battery given by (14) is estimated for comparative purposes. The results show a good fitting of the voltage response (Figure $8(\mathrm{a})$ ). However, the numeric estimation error is still 
quite large up to $80 \%$ (Table 2). Figure 8(b) also illustrates that the impedance of the Randles model with the estimated values given in Table 2 cannot retrieve the true impedance spectra very well. However, compared to the synthetic data, the frequency domain estimation error shows a better performance at low and high frequencies. This might be because of the following reasons, a) there are not enough data points at the frequencies around $1 \mathrm{~Hz}$, and b) due to the measurement noise, the true data are scattered at higher frequencies, around $3 \mathrm{kHz}$.

\section{Conclusions and future work}

In this paper, time-domain fitting of electrochemical impedance models including two resistors and one Constant-Phase-Element (CPE) has been addressed using fractional order system identification methods. The proposed fitting algorithm is based on the simplified refined instrumental variable for continuous-time fractional (srivcf) identification method combined with a gradient-based optimisation to estimate the fractional

order. Both simulation and experimental results demonstrate the effectiveness of the method in estimating the impedance spectra caused by series and charge transfer resistances and double layer capacitance in a real electrochemical cell. However, application of the method to estimate the EIS model parameters using more complex model structures and a wider frequency range is more challenging. One approach could be to decouple a more complex model into simpler models, assuming the range of frequencies covered by each could be separated. As future work, the practical identifiability analysis will be performed to optimally select the data required for the identification. In addition, the effects of temperature and state-of-charge will be added to the EIS model, and the proposed identification method demonstrated in a real application.

\section{Acknowledgements}

This work was funded by the University of Oxford EPSRC Impact Acceleration Account Technology Fund Award EP/K503769/1. This publication benefited from equipment funded by the John Fell Oxford University Press (OUP) Research Fund. We are grateful for the permission of our co-authors from [19] to re-use data, and to Damien Frost and Rob Richardson for assistance with some of the figures.

\section{References}

[1] A. Oustaloup, P. Melchior, P. Lanusse, O. Cois, F. Dancla, The CRONE toolbox for Matlab, IEEE International Symposium on Computer-Aided Control System Design (2000) 190-195.

[2] R. Malti, P. Melchior, P. Lanusse, A. Oustaloup, Towards an Object Oriented CRONE Toolbox for Fractional Differential Systems, IFAC Proceedings Volumes 18 (2011) 10830-10835.

[3] K. S. Cole, R. H. Cole, Dispersion and Absorption in Dielectrics I. Alternating Current Characteristics, The Journal of Chemical Physics 9 (4) (1941) 341. 
[4] J. Randles, Kinetics of rapid electrode reactions, Discuss. Faraday Soc. 1 (1947) 11-19.

[5] J. R. Macdonald, Theory of space-charge polarization and electrode-discharge effects, The Journal of Chemical Physics 58 (11) (1973) 4982-5001.

[6] J. Thevenin, Passivating films on lithium electrodes., Journal of Power Sources 14 (1985) 45-52.

[7] M. Levi, D. Aurbach, Diffusion coefficients of lithium ions during intercalation into graphite derived from the simultaneous measurements and modeling of electrochemical impedance and potentiostatic intermittent intermittent titration characterisation of thin graphite electro, The Journal of Physical Chemistry B 5647 (97) (1997) 4641-4647.

[8] R. R. Richardson, P. T. Ireland, D. A. Howey, Battery internal temperature estimation by combined impedance and surface temperature measurement, Journal of Power Sources 265 (2014) 254-261.

[9] U. Troltzsch, O. Kanoun, H. Trankler, Characterizing aging effects of lithium ion batteries by impedance spectroscopy, Electrochimica Acta 51 (2006) 1664-1672.

[10] E. Barsoukov, J. R. Macdonald, Impedance Spectroscpopy. Theory, Experiment, and Applications, John Wiley and Sons, Inc, 2 edn., 2005.

[11] E. Barsoukov, J. H. Kim, D. H. Kim, K. S. Hwang, C. O. Yoon, H. Lee, Parametric analysis using impedance spectroscopy: relationship between material properties and battery performance, J. New Mat. for Electrochem. Systems 3 (2000) 303-310.

[12] A. M. Dhirde, N. V. Dale, H. Salehfar, M. D. Mann, T. H. Han, Equivalent Electric Circuit Modeling and Performance Analysis of a PEM Fuel Cell Stack Using Impedance Spectroscopy, IEEE Transactions on Energy Conversion 25 (2010) 778-786.

[13] T. Momma, M. Matsunaga, D. Mukoyama, T. Osaka, Ac impedance analysis of lithium ion battery under temperature control, Journal of Power Sources 216 (2012) 304-307.

[14] J.-B. Jorcin, M. E. Orazemb, N. Pébère, B. Tribollet, CPE analysis by local electrochemical impedance spectroscopy, Electrochimica Acta 51 (1) (2006) 1473-1479.

[15] A. Lasia, Modern Aspects of Electrochemistry, R.E. White, B.E. Conway, J.OM. Bockris (Eds.), New York: Kluwer Academic/Plenum, 1999.

[16] W. Waag, D. U. Sauer, Adaptive estimation of the electromotive force of the lithium-ion battery after current interruption for an accurate state-of-charge and capacity determination, Applied Energy 111 (2013) 416-427.

[17] J. Macdonald, J. Schoonman, A. Lehnen, Applicability and power of complex nonlinear least squares for the analysis of impedance and admittance data, Journal of Electroanalytical Chemistry and Interfacial Electrochemistry 131 (1982) 77-95. 
[18] B. Boukamp, A nonlinear least squares fit procedure for analysis of immittance data of electrochemical systems, Solid State Ionics 20 (1986) 31-44.

[19] D. A. Howey, P. D. Mitcheson, V. Yufit, G. J. Offer, N. P. Brandon, On-line measurement of battery impedance using motor controller excitation, IEEE Transactions on Vehicular Technology 63 (6) (2014) $2557-2566$.

[20] G. L. Plett, Extended Kalman filtering for battery management systems of LiPB-based HEV battery packs. Part 1. Background, Journal of Power Sources 134 (2) (2004) 252-261.

[21] D. Andre, M. Meiler, K. Steiner, H. Walz, T. Soczka-Guth, D. Sauer, Characterization of high-power lithium-ion batteries by electrochemical impedance spectroscopy. II: Modelling, Journal of Power Sources $196(12)(2011) 5349-5356$.

[22] C. Birkl, D. Howey, Model identification and parameter estimation for LiFePO4 batteries, Hybrid and Electric Vehicles Conference 2013 (HEVC 2013) (2013) 2.1-2.1.

[23] Y. Hu, S. Yurkovich, Y. Guezennec, B. Yurkovich, Electro-thermal battery model identification for automotive applications, Journal of Power Sources 196 (1) (2011) 449-457.

[24] J. P. Schmidt, P. Berg, M. Schönleber, A. Weber, E. Ivers-Tiffée, The distribution of relaxation times as basis for generalized time-domain models for Li-ion batteries, Journal of Power Sources 221 (2013) $70-77$.

[25] X. Hu, S. Li, H. Peng, A comparative study of equivalent circuit models for Li-ion batteries, Journal of Power Sources 198 (2012) 359-367.

[26] S. K. Rahimian, S. Rayman, R. E. White, Comparison of single particle and equivalent circuit analog models for a lithium-ion cell, Journal of Power Sources 196 (20) (2011) 8450-8462.

[27] P. A. Lindahl, M. A. Cornachione, S. R. Shaw, A Time-Domain Least Squares Approach to Electrochemical Impedance Spectroscopy, IEEE Transactions on Instrumentation and Measurement 61 (2012) 3303-3311.

[28] A. Oustaloup, La dérivation non-entière, Hermès, 1995.

[29] L. Le Lay, Identification fréquentielle et temporelle par modèle non entier, Ph.D. thesis, Université Bordeaux I, Talence, 1998.

[30] J. Lin, Modélisation et identification de systèmes d'ordre non entier, Ph.D. thesis, Université de Poitiers, 2001.

[31] O. Cois, Systèmes linéaires non entiers et identification par modèle non entier : application en thermique, Ph.D. thesis, Université Bordeaux 1, Talence, 2002. 
[32] R. Malti, J. Sabatier, A. H, Thermal modeling and identification of an aluminum rod using fractional calculus, 15th IFAC Symposium on System Identification (SYSID) (2009) 958-963.

[33] J. Sabatier, M. Aoun, A. Oustaloup, G. Grégoire, F. Ragot, P. Roy, Fractional system identification for lead acid battery state of charge estimation, Signal Processing 86 (2006) 2645-2657.

[34] O. Cois, A. Oustaloup, T. Poinot, J.-L. Battaglia, Fractional state variable filter for system identification by fractional model, 6th European Control Conference (ECC2001) (2001) 2481-2486.

[35] P. Young, A. Jakeman, Refined instrumental variable methods of recursive time-series analysis Part I. Single input, single output systems, International Journal of Control 29 (1979) 1-30.

[36] P. Young, Some observations on instrumental variable methods of time-series analysis, International Journal of Control 23 (1976) 593-612.

[37] R. Malti, S. Victor, A. Oustaloup, H. Garnier, Fractional state variable filter for system identification by fractional model, 17th IFAC World Congress (2008) $1-6$.

[38] S. Victor, R. Malti, H. Garnier, A. Oustaloup, Parameter and differentiation order estimation in fractional models, Automatica 49 (4) (2013) 926-935.

[39] P. Young, Recursive estimation and time-series analysis, Springer-Verlag, Berlin, 2 edn., 2011.

[40] A. Raue, C. Kreutz, T. Maiwald, J. Bachmann, M. Schilling, U. Klingmüller, J. Timmer, Structural and practical identifiability analysis of partially observed dynamical models by exploiting the profile likelihood, Bioinformatics 25 (2009) 1923-1929.

[41] M. Rothenberger, J. Anstrom, S. Brennan, H. Fathy, Maximizing Parameter Identifiability of an Equivalent-Circuit Battery Model Using Optimal Periodic Input Shaping, ASME 2014 Dynamic Systems and Control Conference (2014) 1-10.

[42] T. Söderström, P. Stoica, System identification, Prentice-Hall, 1989.

[43] R. L. M. Peeters, B. Hanzon, Symbolic computation of Fisher information matrices for parametrized state-space systems, Automatica 35 (1999) 1059-1071.

[44] R. H. Byrd, J. C. Gilbert, J. Nocedal, A trust region method based on interior point techniques for nonlinear programming, Mathematical Programming, Series B 89 (2000) 149-185.

[45] EC-Lab User Manual v10.37, Bio-Logic Science Instruments, 2014. 


\section{List of Figures}

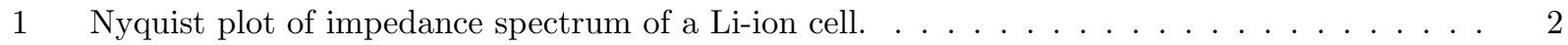

2 Battery EIS equivalent circuit model $(2) \ldots \ldots \ldots \ldots \ldots$

3 Evaluation of the proposed algorithm using synthetic data: measurement signals. . . . . . . 8

4 Evaluation of the proposed algorithm using synthetic data: the cost function $J$ given by (12) versus $\alpha$. The minimum $J$ is obtained at the best estimation of the fractional exponent $\alpha . \ldots 9$

$5 \quad$ Evaluation of the fractional and Randles models using the synthetic data given in Table 1. . 10

6 Diagram of voltage and current measurement circuit. . . . . . . . . . . . . . . . 11

$7 \quad$ Evaluation of the proposed algorithm using real data: filtered signals. . . . . . . . . . . . 11

8 Evaluation of the fractional and Randles models using the real data given in Table 2 . . . . 12 